\title{
Design of Toll Plaza Based on Genetic Algorithm
}

\author{
Jia Feng ${ }^{a}$, Wei Xiang ${ }^{b, *}$ \\ College of Electric and Information Engineering, Southwest University for Nationalities, Chengdu \\ 610041, China \\ a1987523925@qq.com, b3730544@qq.com, \\ Corresponding author: Wei Xiang
}

\begin{abstract}
Keywords: Toll Plaza, Genetic Algorithm, Optimal Value.
\end{abstract}
\begin{abstract}
This paper studies the design of toll plaza based on genetic algorithm. Based on the idea of the function value of the genetic algorithm, the area of the toll station and the traffic accident rate are taken as the objective function, to find out the optimal value of the construction cost under the influence of the number of toll stations, the gradient rate, the width of the toll station.
\end{abstract}

\section{Introduction}

With the rapid development of the expressway, as an important part of the highway toll station building engineering is becoming more and more important.Toll station is the bottleneck of the highway traffic, it's operation make a direct impact on the road capacity, but also an important part of the planning and construction of toll stations. Therefore, to solve the congestion problem of toll station is the basis of the design of toll station and economic evaluation.In the highway toll station design, not only to consider the smoothness of the highway, and secondly, the shape of the toll station area is not to be underestimated.In this paper, a genetic algorithm is used to establish the model of smooth and reasonable cost, determining the optimal solution of toll station.

\section{Genetic Algorithm}

Genetic algorithm is a simulation of the evolution of organisms, start from an initial population, repeated selection, crossover and mutation operations, a set of candidate solutions is retained in each iteration.

According to some specified index, the evolution of population is getting closer and closer to a certain goal, so as to obtain the optimal solution or satisfactory solution. The genetic algorithm uses the vicissitudes rule of probability to guide the search direction, searching from string set,the coverage is large, which is favorable to the global optimization and reduces the risk of being trapped in the local optimal solution. At the same time, the algorithm is easy to be parallelized, and only the fitness function value is used to evaluate the individual.On this basis, genetic manipulation is to be used.

\section{The Establishment of The Model}

\subsection{Model Establishment}

We will divide charge station into three parts, respectively is the entry section, waiting for the service period, leaving the toll stations, as shown in Fig.1.

In the design of toll station, based on the impact of construction costs and traffic fluency. The most important thing is the design of the toll area.

The structure of the toll station is built in the form of figure one, the structure of fan in and fan out area is basically the same. And the length of the intermediate service area of each toll station is the same as that of the national road construction law.Therefore, this article only take the fan area of the charging station into to consider. The shape of the entrance area of the toll station, as shown in Fig.2. 


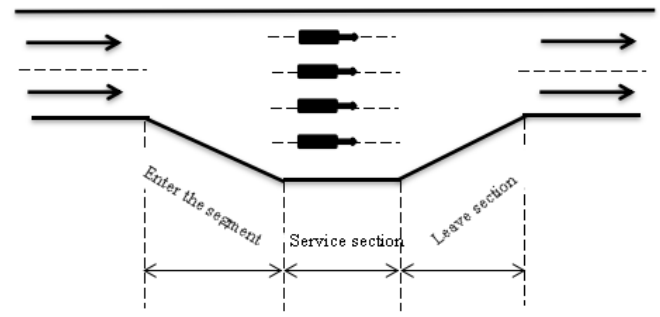

Figure 1. Toll station overall map

3.1.1 Rate of change of toll plaza

Rate of change of toll plaza:

$$
\phi=\tan \alpha
$$

According to the literature search, get the functional relationship between the rate of change of toll plaza and the number of traffic accidents ${ }^{[1]}$

$$
Y=1.4231 e^{8.0664 \phi}
$$

In the formula, Y-the number of traffic accidents corresponding to the gradual change rate of toll

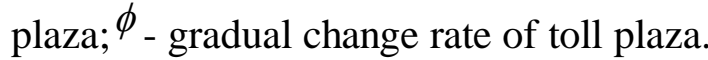

Matlab mapping can be used to clearly know the relationship between the two, as shown in Fig.3.

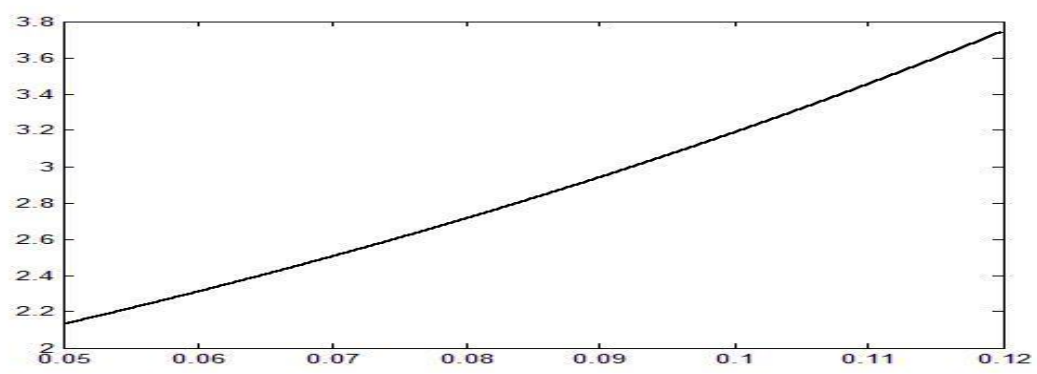

Figure 3. Relationship between gradual change rate and traffic accidents

It can be seen from the graph that the larger the gradient rate is, the higher the frequency of traffic accidents.

3.1.2 Calculation of area of fan entrance

According to the geometric relation in Figure two, the area of the fan area can be expressed as

$$
w_{f}-w_{u}=\phi^{*} l_{n}
$$

The area of the entrance area of the toll lane:

$$
s=l_{n} * w_{f}-\frac{1}{2} * \phi^{*} l_{n}^{2}
$$

Specifies the width of each toll station (including toll booths and toll lanes)is $d$,

$$
w_{f}=d^{*} n
$$

According to determination of the number of toll stations, the value of toll lane can be calculated,

$$
l_{n}=n a_{l}
$$

The coefficients of $a_{l}$ used in the calculation of $l_{n}$, values are generally taken from 0.01 to 0.025 , so take 0.02 kilometer.

The area of the entrance area of the toll lane:

$$
s=n a_{t} * d * n-\frac{1}{2} * \phi *\left(n a_{t}\right)^{2}
$$

\subsection{Highway Toll Plaza Design Specification Parameters}

According to the national highway toll plaza design specifications, we can determine the local spatial range, as shown in Table 1. 
Table 1. Design parameters of Expressway Toll Plaza

\begin{tabular}{|c|c|c|}
\hline Parameter Name & Value Range & Company \\
\hline Channel width $d_{1}$ & $2.8 \sim 4.5$ & $m$ \\
\hline Toll island width $d_{2}$ & $2.0 \sim 2.7$ & $m$ \\
\hline Number of toll stations $n$ & $2 \leq \mathrm{n} \leq 8$ & unit \\
\hline Gradual change rate $\phi$ & $1 / 7 \sim 1 / 3$ & \\
\hline
\end{tabular}

\section{To Solve the Optimal Value}

\subsection{Limit to the Optimal Value}

In the functional relationship, if we seek the optimal value of multivariate function, we often use the left and right limits of the function thought to find the optimal solution ${ }^{[2]}$.Below we will use the left and right limit to find the optimal solution.

According to equation (7) we know that $\mathrm{s}$ is a multivariate function for $\mathrm{n}, \phi$ and $\mathrm{d}$, in order that the $\mathrm{s}$ has a minimum value, we should make $\mathrm{s}$ to get the minimum $s_{\min }$. we should make the aforesaid $20 d n^{2}$ minium and consequent $200 \phi n^{2}$ maximum, the width of per unit of the toll station back is $4.8, \phi$ is $1 / 3$. Similarly, to get $\mathrm{s}$ has a maximum value, we should make the maximum of aforesaid, minimum of consequent, the width of per unit of the toll station back is $7.2, \phi$ is $1 / 20$. The maximum s value of solution is $s_{\max }=115.4 n^{2}$. Toll station construction area and the number of toll island into a non-linear relationship. According to state regulations, when $\mathrm{n}$ is two, $106.8 \leq s \leq 461.6$. When $\mathrm{n}$ is eight, $1708.8 \leq s \leq 7385.6$

\subsection{Establishment of Genetic Algorithm Parameters}

Formula (7) is a multivariate function, which can be seen that the fan area is related to many factors. When solving the fan in the area, the cost based on the national land acquisition is higher than the construction of charging platform and the cost of paving, we mainly consider the minimum cost of the land area. This paper puts forward the optimal genetic algorithm is used to solve the fan in the area of value, toll station number is $\mathrm{n}$, the width of each toll station (including toll booths and toll lane width) is $\mathrm{d}$, gradual change ratio are served as three genes on the chromosome.

First of all, coding the individual gene. The independent variables of the function can be regarded as the representation form of the genetic algorithm, and the binary mapping is used to map the phenotype to genotype. If the minimum accuracy of the results is not less than 0.001 , the binary string of each variable can be taken up to 10 bits. Because the objective function is a function of three variables, so there are three genes on each chromosome. The length of each gene is 10 , so the total length of a single chromosome is 30 , at this point, a $100 * 3$ matrix is generated. We can produce a certain number of individuals to form a population, the size of the population is the number of individuals in the population. Secondly, to determine the fitness of this genetic operation. In this paper, the s of the objective function value is used as the fitness, the maximum of the construction area is the maximum of the objective function, and the fitness is higher. Then, select the better individual. For the individual selection we use the roulette selection method ${ }^{[3]}$. This algorithm is simple, in order to select mating individuals, it is necessary to carry out multiple rounds of selection, each round to produce a $[0,1]$ uniform random number, the random number as a selection pointer to determine the selected individual. Finally, genetic manipulation are taken out. An individual represented by a natural binary encoding method $^{[4]}$.If the cross variable using the gray code for encoding the individual, if there is a bit difference between the string, the corresponding parameter value is only doing a small difference.

\section{Matlab Simulation}

According to the above algorithm programming under the MATLAB environment, the simulation waveform is shown in Fig.4. 


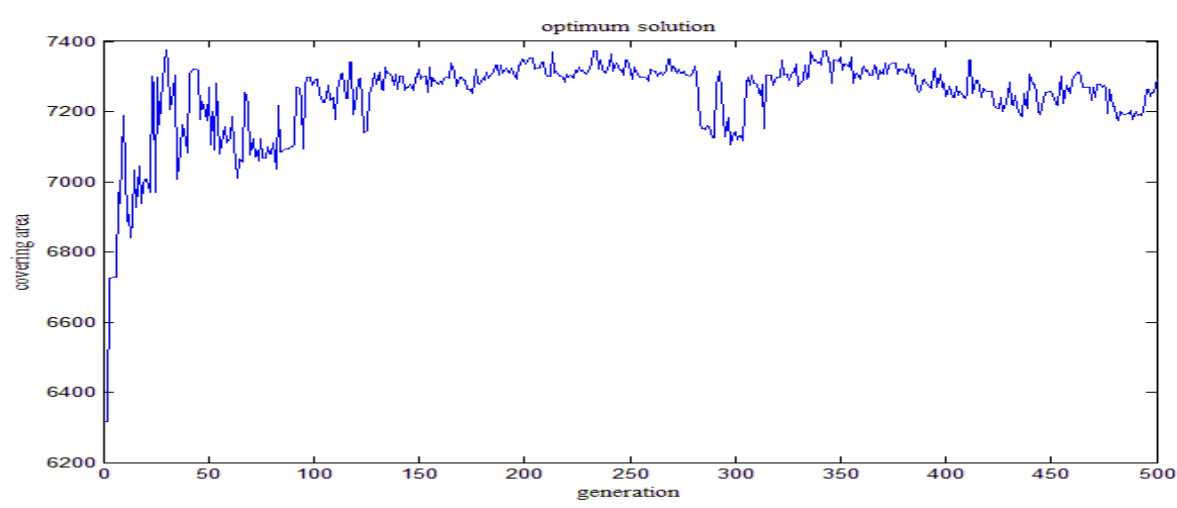

Figure 4 optimal value of construction area

From the above chart shows, with the genetic operation, the construction area of the toll station in the fluctuation of a determined value near, does not appear large variation values, through genetic algorithm to find the optimal value of 7376.66, as the value of the solution. When the optimal solution is obtained by $\mathrm{s}$, the optimal value of each parameter is shown in Table 2.

Table 2. Optimal parameters

\begin{tabular}{|c|c|c|c|}
\hline Parameter Name & $\begin{array}{c}\text { Optimal Solution of Function } \\
\text { Relation }\end{array}$ & $\begin{array}{c}\text { Genetic Algorithm } \\
\text { Optimal Solution }\end{array}$ & Unit \\
\hline Construction area & $106.8 \leq s \leq 7385.6$ & 7376.66 & $m^{2}$ \\
\hline Toll station width $d$ & $4.8 \sim 7.2$ & 6.6440 & $m$ \\
\hline $\begin{array}{c}\text { Toll station } \\
\text { platform } n\end{array}$ & $2 \leq \mathrm{n} \leq 8$ & 7.6305 & \\
\hline Gradual change rate $\phi$ & $1 / 7 \sim 1 / 3$ & 0.2186 & case \\
\hline Traffic accident & $4.505 \sim 20.940$ & 8.299 & \\
\hline
\end{tabular}

From the above table, the optimal solution obtained by genetic algorithm are in the range of the optimal solution, and the optimal solution is not the estimation range, but the exact value in the case of the smallest error, has more practical reference value to the implementation of the project, further proof of the superiority of the algorithm in this paper.

\section{Conclusion}

In this paper, the genetic algorithm is used to estimate the area of the toll station and the traffic accident rate, the superiority of genetic algorithm and the rationality of the algorithm are proved by Matlab simulation, and make an effective assessment to the number of toll stations, gradient rate, toll station width and other factors.

\section{Acknowledgments}

This work was partially supported by National Nature Science Foundation (61673016), SWUN Construction Projects for Graduate Degree Programs (2017XWD-S0805), Advance Research Pro-gram of Electronic Science and Technology National Program(2017YYGZS16) and Sichuan Youth Science and technology innovation research team (2017TD0028), also was financially supported by students innovation training project of SWUN(S201610656077).

\section{References}

[1] Zhang Min,Chen Hong,Wu Xiaowu . Safety evaluation model of expressway toll station [J]. Chinese Journal of safety science, 2009, (): 139-144+179.

[2] Wang Shengjun. Application of function idea in the problem of parameter estimation [J]. science and Technology Innovation Herald, 2016, (20): 166+168.

[3] Zhang Chen, Zhan Zhihui. Comparison of selection strategies for genetic algorithms [J]. computer 
engineering and design, 2009, (): 5471-5474+5478.

[4] Yang Xiaohua, Lu Guihua, Yang Zhifeng, Li Jian Qiang. Gray coding genetic algorithm and theory research [J]. systems engineering theory \& practice, 2003, (03): 100-106. 